\title{
Multi-Verse Algorithm based Approach for Multi- criteria Path Planning of Unmanned Aerial Vehicles
}

\author{
Raja Jarray $^{1}$, Soufiene Bouallègue ${ }^{1,2}$ \\ Research Laboratory in Automatic Control (LARA) \\ National Engineering School of Tunis (ENIT) \\ University of Tunis EL MANAR, BP 37, Le Belvédère, 1002 Tunis, Tunisia ${ }^{1}$ \\ Higher Institute of Industrial Systems of Gabès (ISSIG), 6011 Gabès, Tunisia ${ }^{2}$
}

\begin{abstract}
In this paper, a method based on a Multiobjective Multi-Verse Optimizer (MOMVO) is proposed and successfully implemented to solve the unmanned aerial vehicles' path planning problem. The generation of each coordinate of the aircraft is reformulated as a multiobjective optimization problem under operational constraints. The shortest and smoothest path by avoiding all obstacles and threats is the solution of such a hard optimization problem. A set of competitive metaheuristics such as Multiobjective Salp Swarm Algorithm (MSSA), Grey Wolf Optimizer (MOGWO), Particle Swarm Optimization (MOPSO) and Non-dominated Sorting Genetic Algorithm II (NSGA-II) are retained as comparison tools for the problem's resolution. To assess the performance of the reported algorithms and conclude about their effectiveness, an empirical study is firstly performed for solving different multiobjective test functions from the literature. These algorithms are then used to obtain a set of optimal Pareto solutions for the multi-criteria path planning problem. An efficient Technique for Order of Preference by Similarity to Ideal Solution (TOPSIS) of Multi Criteria DecisionMaking (MCDM) model is investigated to find the optimal solution from the non-dominant ones. Demonstrative results and statistical analysis are presented and compared in order to show the effectiveness of the proposed MOMVO-based path planning technique.
\end{abstract}

Keywords-Unmanned aerial vehicles; path planning problem; multiobjective optimization; multiobjective multi-verse algorithm; decision-making model; nonparametric statistical tests

\section{INTRODUCTION}

The Unmanned Aerial Vehicles (UAVs) have shown their commitment in various military and civil applications [1, 2]. The problem of paths planning, especially within a flight environment with threats and obstacles, is one of the key elements in the framework of design and control of these aerial robots. Such a complex problem can be seen and treated as a hard optimization problem under operational constraints [3][15]. The resolution of such a problem allows the unmanned aircraft to autonomously calculate the optimal or almost optimal path from the starting point to the target, based on the requirements and constraints of the activity.

In the literature, various approaches and techniques have been proposed to solve such kind of complex optimization problems. The graph-based techniques are extensively adopted and show some effective advantages. The well-known Voronoi diagram searching method [3], rapidly-exploring random graph algorithm [4], A* algorithm [5], D* Lite approach [6] and artificial potential field algorithm [7] are the main used approaches. Often, it is difficult to consider the movement limitations of the UAVs in these types of planning methods, which means that they cannot normally be used within practical situations [8]. Another category of path planning methods, such as the population-based scalable algorithms, can overcome these shortcomings. As an example, the authors in [9] used the Genetic Algorithm (GA) to solve the shortest path problem in order to scan large agricultural lands and collect data. The authors in [10] developed an algorithm that uses the well-known Particle Swarm Optimization (PSO) method to solve the trajectory planning problem for multiple UAVs in a receding horizon framework. In [11], a two new hybrid metaheuristics that combine the PSO method both with the genetic algorithm and harmony search algorithm have been proposed to solve the UAVs' path planning problem. In [12], the authors have solved the UAVs' path planning problem based on a recent global metaheuristic named Grey Wolf Optimization (GWO). In [13], an improved Ant Colony Optimization (ACO) algorithm has been given by introducing the metropolis criterion into the node filtering mechanism in order to generate the initial trajectory and avoid the risk of falling into the optimal local solution and stagnation. In [14], a modified central force optimization based method has been introduced to address the rotary wing vertical take-off and landing aircraft trajectory planning. The authors in [15] have presented a 3D path planning algorithm based on an adaptive sensitivity decision operator associated with a PSO method.

In addition, most real path planning problems need to be solved by considering different conflicting goals such as price and quality. The conflicting objectives must be addressed simultaneously and the weighted based methods are usually used [14, 16]. Nevertheless, it is difficult to determine the relationship between the weighting factors. Therefore, these objectives should be treated by multiobjective metaheuristics which are applied in many others domains $[17,18]$. The idea of using multiobjective optimization concepts for path planning problem formulation and resolution seems a promising solution and it has been exploited in this work.

In [19], a Multiobjective Genetic Algorithm (MOGA) based method has been used to solve the complex path planning problems implying a mission of UAVs and a set of ground control stations. Many objectives have been optimized such as the makespan, the fuel consumption, and covered distance. In [20], the authors developed an improved 
Multiobjective Particle Swarm Optimization (MOPSO) algorithm to find collision-free and feasible paths with various minimum factors such as altitude, length and angle variable rate. The authors in [21] have improved a Non-dominated Sorting Genetic Algorithm III (NSGA-III) by adding adaptive genetic operators in the offspring population generation to solve the path planning problems. In [22], an improved multiobjective ACO algorithm has been adopted in which the objective function for optimization is formulated to make UAV drone following a short, safe and smooth path. Such an algorithm assumes that the environment is known in advance. The authors in [23] have used a safety index map (SIM) to catch obstacles in the geography map. Then, a multiobjective path planning approach based on a Crowding Distance NSGAII (CDNSAG-II) metaheuristic is proposed while considering both path length and safety as the main flight objectives. In [24], the path planning problem has been modeled as a problem with high complexity involving several tasks. Such a modeling approach presented high convergence rate for multiobjective solvers. The authors have used a weighted random generator that can concentrate the search on potentially better regions of the solution space to reduce the convergence rate of the used Multiobjective Evolutionary Algorithm (MOEA) solver. The authors in [25] have solved a multi-UAVs' trajectory planning problem using the concepts of MOPSO metaheuristic.

Based on the aforementioned studies, and regarding the drawbacks of the cited methods especially in terms of complexity and time consuming, the main contribution of this paper is the development of a novel strategy of reformulation and solving of a multi-criteria path planning problem under operational constraints based on a recent and unified MOMVO algorithm. The proposed MOMVO-based method allows the UAVs to autonomously calculate the optimum or near optimal path from the starting point to the target while avoiding all threats and obstacles considered in the flight environment. The choice of a solution among all the optimal Pareto ones requires a higher-level decision-making approach. The Technique for Order of Preference by Similarity to Ideal Solution (TOPSIS) is proposed for this path planning methodology. The demonstrative results are presented, statistically analyzed and compared with each other as well as with those obtained by the competitive MOPSO, NSGA-II, MSSA and MOGWO algorithms.

The reminder of this paper is organized as follows. In Section II, the flight environment is topologically modeled and a multiobjective reformulation of the UAVs' path planning problem is derived. This section is ended by the description of the proposed offline path planning algorithm. Section III presents the description of the proposed multiobjective multiverse algorithm MOMVO as well as its adaptation to solve the reformulated multi-criteria path planning problem. A pseudocode of such a multiobjective algorithm is given for the soft implementation. In Section IV, numerical simulations are carried out and discussed to show the effectiveness of the proposed MOMVO-based path planning approach. Several comparisons with a set of competitive algorithms are made through this study. Section V concludes the paper.

\section{PAth PlanNing Problem Formulation}

\section{A. Flight Environment Modeling}

In robotics, the path planning procedure is the creation of a plan to guide a UAV, similar to a moving object in the space, from the starting point $\mathrm{S}$ to the destination one $\mathrm{P}$ with the spatial coordinates denoted as $\left(x_{S}, y_{S}, z_{S}\right)=\left(x_{1}, y_{1}, z_{1}\right)$ and $\left(x_{P}, y_{P}, z_{P}\right)=\left(x_{n}, y_{n}, z_{n}\right)$, respectively. The navigation model used in this study is shown in Fig. 1. In a real navigation environment, it is very challenging to define the geometric coordinates of the obstacles and threats of the UAV drone. In this work, a danger zone is characterized by a cylinder model supposed to be static in the 3D flight environment as depicted in Fig. 2.

The $\mathrm{x}$-axis range of the flight space is divided into $n-1$ equal segments which are denoted as $x_{1}, x_{2}, x_{3}, \ldots, x_{n}$. The perpendicular planes $\left(L_{1}, L_{2}, L_{3}, \ldots, L_{n}\right)$ are passed by these corresponding division points. By taking a waypoint $\boldsymbol{w}_{i}=\left(x_{i}, y_{i}, z_{i}\right)$ at each plane $L_{i}$ and forming a waypoints' sequence $\boldsymbol{W}=\left\{S,\left(x_{2}, y_{2}, z_{2}\right), \ldots,\left(x_{n-1}, y_{n-1}, z_{n-1}\right), P\right\}$, a flight path is then generated by connecting all these waypoints. In this study, the problem of path planning is solved by optimizing the series of the waypoints in order to generate a shorter and smoother path from the starting point $S$ to the goal point $\mathrm{P}$ while avoiding the existing obstacles and threats. Based on the cubic Spline interpolation method, these waypoints are connected to obtain the desired smooth path. In this path's modeling strategy, the $x$-coordinates of all waypoints are know in advance but those of the $\mathrm{y}$ - and $\mathrm{z}$-axis have to be determined. Subsequently, the generation of each coordinate waypoint $\boldsymbol{w}_{i}=\left(x_{i}=x_{\text {known }}, y_{i}, z_{i}\right) \quad, \quad i=2,3, \ldots, n-1, \quad$ is formulated as a multiobjective optimization problem with the decision variables $\boldsymbol{\theta}=\left\{y_{i}, z_{i}\right\}$ and under operational nonlinear and complex constraints. In this mathematical formulation, the variables $y_{i}$ and $z_{i}$ denote the $\mathrm{y}$ - and z-coordinates of the ith waypoint, respectively.

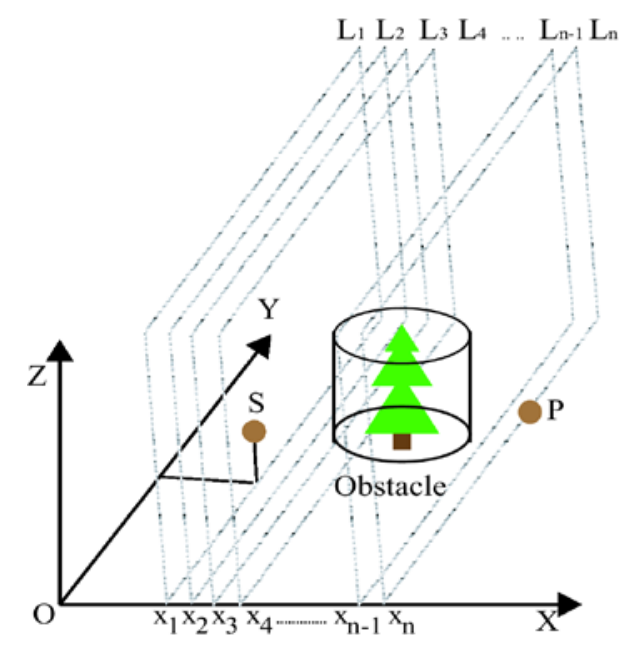

Fig. 1. Geometry of the UAV's Navigation Space. 


\section{B. Problem Formulation}

In the mathematical multiobjective optimization formalism, a generic constrained problem is defined as follows [26]:

$$
\left\{\begin{array}{l}
\underset{\boldsymbol{\theta} \in \boldsymbol{D} \subseteq \square^{q}}{\operatorname{Minimize}} F(\boldsymbol{\theta})=\left\{f_{1}(\boldsymbol{\theta}), f_{2}(\boldsymbol{\theta}), \ldots f_{M}(\boldsymbol{\theta})\right\} \\
\text { s.t: } \\
g_{v}(\boldsymbol{\theta}) \leq 0 \quad v=1,2, \ldots, V \\
h_{w}(\boldsymbol{\theta})=0 \quad w=1,2, \ldots, W \\
\boldsymbol{\theta} \in \boldsymbol{D} \subseteq \mathrm{R}^{q}
\end{array}\right.
$$

where $f_{m}: \square^{q} \rightarrow \square \quad$, for $m=1,2, \ldots, M$, denote the objective functions to be minimized, $\boldsymbol{D}=\left\{\boldsymbol{\theta} \in \square^{q}, \boldsymbol{\theta}_{\min } \leq \boldsymbol{\theta} \leq \boldsymbol{\theta}_{\max }\right\}$ is the bounded search domain, $g_{v}: \square^{q} \rightarrow \square$ and $h_{w}: \square^{q} \rightarrow \square$ are the inequality and equality constraints, respectively, $q \in \mathrm{N}$ is the dimension of the optimization problem, i.e. the number of decision variables.

For the UAVs' navigation process, the length of the planned path is very important in the case of several missions. A shorter path can extend the life of an UAV and remains desirable in all planning problems. The criteria which can be considered for the path planning process are especially related to the path length and drone's attitude. According to this flight specification and for a given $\mathrm{i}^{\text {th }}$ waypoint, the related objective function to be minimized in problem (1) can be formulated as follows:

$$
\begin{aligned}
f_{1, i}(\boldsymbol{\theta}) & =\sqrt{\left(x_{i}-x_{i-1}\right)^{2}+\left(y_{i}-y_{i-1}\right)^{2}+\left(z_{i}-z_{i-1}\right)^{2}} \\
& +\sqrt{\left(x_{i}-x_{n}\right)^{2}+\left(y_{i}-y_{n}\right)^{2}+\left(z_{i}-z_{n}\right)^{2}}
\end{aligned}
$$

where $\left(x_{n}, y_{n}, z_{n}\right)$ denotes the coordinates of the destination point $\mathrm{P}, \boldsymbol{n}$ is the number of the waypoints and $\boldsymbol{\theta}=\left\{y_{i}, z_{i}\right\}$ are the unknown $\mathrm{y}$ - and $\mathrm{z}$-axis coordinates of the given $\mathrm{i}^{\text {th }}$ waypoint, respectively.

In addition, the dynamical characteristics of a given UAV cannot be completely ignored. In order to limit the straightness of the path, the angles between two adjacent segments' $\vec{\varphi}$ and $\vec{\psi}$ are introduced. This path planning specification is modeled by the following cost function:

$$
f_{2, i}(\boldsymbol{\theta})=\arccos \left(\frac{\vec{\varphi} \vec{\psi}}{|\vec{\varphi}||\vec{\psi}|}\right)
$$

where $\vec{\varphi}$ means $\left(x_{i-2}-x_{i-1}, y_{i-2}-y_{i-1}, z_{i-2}-z_{i-1}\right)$ and $\vec{\psi}$ denotes $\left(x_{i}-x_{i-1}, y_{i}-y_{i-1}, z_{i}-z_{i-1}\right)$.

The collision avoidance is essential for any path planning task. In its own navigation process, a drone cannot in any case cross the threat zones and/or fly over them in order to avoid the risk of being detected by the radars or missiles within a military application. Such an avoidance specification is modeled by the following family of nonlinear and hard constraints:

$$
g_{1, i}(\boldsymbol{\theta})=r_{t}+\delta_{\min }-\sqrt{\left(x_{i}-x_{t}\right)^{2}+\left(y_{i}-y_{t}\right)^{2}} \leq 0
$$

where $\left(x_{t}, y_{t}, r_{t}\right)$ is the coordinates of the static threat zone, $\left(x_{t}, y_{t}\right)$ presents the center on the XOY flight plan, $r_{t}$ is the radius of a given obstacle and $\delta_{\min }$ is the safety distance defined as shown in Fig. 2.

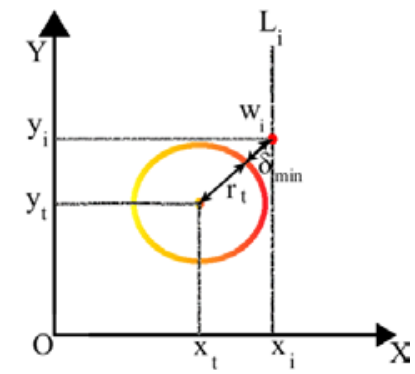

Fig. 2. Illustration of the Threat Zones in Flight Space.

Considering all these defined objectives and constraints, the formulated multiobjective optimization problem for the UAV's path planning according to a given ith waypoint is defined as follows:

$$
\left\{\begin{array}{l}
\underset{\boldsymbol{\theta} \in \boldsymbol{D} \subseteq \square^{2}}{\operatorname{Minimize}} F(\boldsymbol{\theta})=\left\{f_{1, i}(\boldsymbol{\theta}), f_{2, i}(\boldsymbol{\theta})\right\} \\
\text { s.t : } \\
g_{1, i}(\boldsymbol{\theta}) \leq 0 \\
\boldsymbol{\theta} \in \boldsymbol{D} \subseteq \mathrm{R}^{2}
\end{array}\right.
$$

where $f_{1, i}(),. f_{2, i}($.$) and g_{1, i}($.$) are the cost and$ constraint functions given in (2), (3) and (4), respectively, $\boldsymbol{\theta}=\left\{y_{i}, z_{i}\right\}$ is the decision variable of the problem.

To handle with the operational constraints of problem (5), the following static penalty function is used [27]:

$\phi_{m, i}(\theta)=f_{m, i}(\theta)+\sum_{v=1}^{v} \lambda_{v, i} \max \left\{0, g_{v, i}(\theta)\right\}^{2}$

where $\lambda_{v} \in \square^{+}$is the weighting coefficient associated to the $\mathrm{v}^{\text {th }}$ constraint, $V$ is the total number of the inequality types of constraints and $m=1,2, \ldots, M$.

\section{Proposed Planning Procedure}

In the multiobjective optimization framework, many methods have been developed for the selection of an optimal solution from a given set of Pareto non-dominated ones [28, 29]. In this work, the technique for order of preference by similarity to ideal solution TOPSIS is used to make decision about the optimal solution for problem (5). Algorithm 1 is so proposed for the complete resolution of the UAV's path planning problem (5). 


\section{Algorithm 1: offline path planning algorithm}

\section{Step 1: Initialization}

Initialize the starting point $\left(x_{1}, y_{1}, z_{1}\right)$, the destination point $\left(x_{n}, y_{n}, z_{n}\right)$, the waypoints matrix $P=\left[x_{1} ; y_{1} ; z_{1}\right]$. Divide the $x-$ axis range into $n-1$ equal portions and set the vector

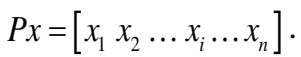

\section{Step 2: Generation of the waypoints}

For $i=2$ to length $|P x|-1$ do

A multiobjective optimizer is used to obtain a set of optimal Pareto solutions of problem (5).

A multi-criteria decision making method, i.e. TOPSIS, is used to find the optimal solution.

A new waypoint is generated $P_{\text {new }}=\left[P x(i) ; y_{\text {opt }} ; z_{\text {opt }}\right]$.

$P=\left[\begin{array}{ll}P & P_{\text {new }}\end{array}\right]$.

\section{End For}

Step 3: Interpolation of the waypoints

Waypoints are linked by a cubic spline interpolation method.

\section{Proposed Multiobjective Multi-Verse Optimizer}

\section{A. Basic Concepts}

The Multi-Verse Optimizer (MVO), originally proposed by Mirjalili et al. [30], is a recent global metaheuristic based on the physics theories about the existence of multi-verse. The interaction among different universes is ensured based on the concepts of white/black holes and worm holes.

The optimization process of the MVO metaheuristic begins with a set of randomly solutions. At each step, the objects from one universe (decision variables) move according to their inflation rates (fitness values) to another via the white/black holes, and displace within a universe or to another via a worm hole. In this process, the white/black holes are used for the improvements of the exploration mechanism, while the worm holes are employed for the exploitation one. The main updating equations in the MVO metaheuristic are given as follows [30]:

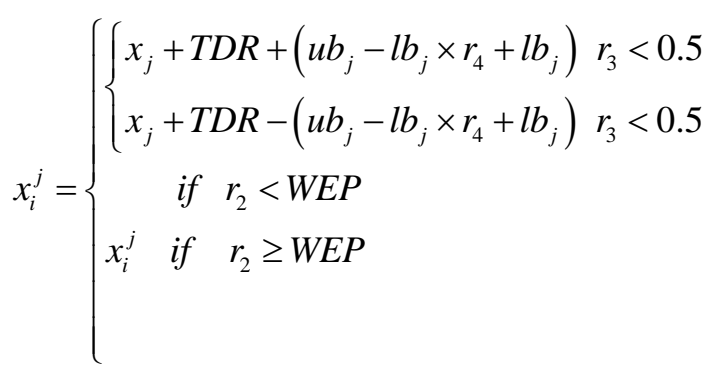

where $x_{i}^{j}$ denotes the $\mathrm{j}^{\text {th }}$ component in the $\mathrm{i}^{\text {th }}$ solution, $x_{j}$ indicates the $\mathrm{j}^{\text {th }}$ variable of the best universe, $l b_{j}$ and $u b_{j}$ are the lower and upper bounds, respectively, $r_{2}, r_{3}$ and $r_{4}$ are random numbers defined in the interval $[0,1], T D R$ and WEP present the travelling distance rate and the worm hole existence probability, respectively. They are defined as follows [30]:
$W E P=\min \_$pro + iter $\left(\frac{\max _{-} \text {pro }- \text { min_p }_{-} \text {pro }}{\text { Max_iter }}\right)$

$T D R=1-\frac{(\text { iter })^{\frac{1}{p}}}{\text { Max_iter }}$

where min_pro and max_pro are the minimum and maximum probability of the wormhole existence, respectively, iter is the current iteration, Max_iter is the maximum number of iterations and $p$ is the exploitation accuracy.

In order to elaborate a multiobjective version of the MVO metaheuristic for problem (5), a concept of the archive is added to the research mechanism in a similar way with the wellknown literature approaches [31]. Like the MVO, the solutions of the MOMVO algorithm are enhanced using black, white and worm holes. For selecting solutions from the archive, the leader selection method is implemented to establish tunnels among solutions. A roulette wheel approach is used to select the fittest solutions. Obviously, a limited number of solutions can be accommodated in the archive. In order to remove the unsatisfactory ones, a probabilistic mechanism given by Equation (10) is proposed as follows [31]:

$\delta_{i}=N_{i} / C$

where $N_{i}$ defines the number of the vicinity solutions and $\mathrm{c}$ is a constant which is greater than 1 .

\section{B. Pseudo-Code}

According to the above evolution equations (7)-(10) and the basic concept of the MOMVO algorithm, a pseudo-code for its software implementation is presented in Algorithm 2.

\section{Algorithm 2: MOMVO}

Step 1: Set the parameters of the MOMVO algorithm

Step 2: Randomly initialize the positions of universes.

Step 3: While $($ iter $<$ Max_iter +1$)$ do

Update WEP and TDR by applying Eq. (8) and Eq. (9).

For each universe do

Boundary checking for the universes inside search space.

End For

Calculate the inflation rate (fitness) of universes.

Sort fitness values.

Find the non-dominated solutions.

Normalize the inflation rates of each universe.

Update the archive.

If the archive is full do

Delete some solutions from the archive.

\section{End If}

Update the position of universes according to Eq. (7)

If any new solution of the archive is outside boundaries do

End If

Update the boundaries to cover the new solution(s).

Increment iter

Step 4: Stop the algorithm when it reaches Max_iter . 


\section{Simulation RESUlts AND Discussion}

\section{A. Numerical Validation on CEC'2009 Test Suite}

In order to evaluate the performance of the reported competitive algorithms MSSA, MOMVO, MOGWO, NSGA-II and MOPSO for problem (5), an empirical study is firstly conducted based on a benchmark of 9 standard multiobjective test problems from the CEC'2009 test suite [32]. The main control parameters of the reported solvers are setting as follows:

- MOMVO [31]: min and max of wormhole existence probability: 0.2 and 1 , respectively.

- MSSA [33]: without control parameters.

- MOGWO [34]: grid inflation 0.1, number of grids per each dimension 10, leader selection pressure 4 and extra repository member selection pressure 2 .

- NSGA-II [35]: crossover probability 0.7, mutation probability 0.4 and mutation rate 0.02 .

- MOPSO [36]: social and cognitive parameters 2, grid inflation 0.1 , leader selection pressure parameter 2 and number of grids per each dimension 7.

In order to have a fair comparison, the termination criterion of all competitive algorithms is set as a maximum number of iterations reached 100 . The population size is fixed as 50 . After numerical experimentations on a PC with i7 Core 2 Duo/2.67 $\mathrm{GHz} \mathrm{CPU}$ and 6.00 GB RAM, the obtained optimization results show the effectiveness of all reported algorithms with a remarkable superiority of the proposed MOMVO algorithm in terms of convergence fastness and solutions' quality. The obtained Pareto fronts are closer to the well-known ones with satisfied distribution and repartition of solutions.

\section{B. Path Planning Problem Resolution}

In this subsection, the effectiveness and validity of the proposed MOMVO-based planning approach are presented and compared to those of the reported competitive algorithms in different flight scenarios as given in Table I. In order to have an equitable comparison, the population size retained for all reported algorithms in the resolution of problem (5) is set as 100 and the maximum number of iterations is equal to 100 . Path planning problems involve finding a feasible path from the starting point to the target one by avoiding out all the obstacles and threats. In this work, five experimental scenarios are investigated. Each of them is specified by the number and position of the static threats as shown in Table I.

TABLE I. INFORMATION ON EXTERNAL INSTALLATIONS OF THE FLIGHT ENVIRONMENT

\begin{tabular}{|l|l|l|l|}
\hline Scenarios & $\begin{array}{l}\text { Starting point } \\
{[\mathbf{k m}]}\end{array}$ & $\begin{array}{l}\text { Destination point } \\
{[\mathbf{k m}]}\end{array}$ & $\begin{array}{l}\text { Threats' } \\
\text { number }\end{array}$ \\
\hline 1 & {$[2,2,0]$} & {$[8,10,0]$} & 5 \\
\hline 2 & {$[1,2,0]$} & {$[10,10,0]$} & 7 \\
\hline 3 & {$[1,10,0]$} & {$[15,2,0]$} & 10 \\
\hline 4 & {$[4,4,0]$} & {$[19,13,0]$} & 12 \\
\hline 5 & {$[1,18,0]$} & {$[17,4,0]$} & 15 \\
\hline
\end{tabular}

Since the generation of each waypoint of the flight path is considered as a solution of the formulated multiobjective optimization problem (5), all reported algorithms are executed on such a constrained problem and the obtained Pareto fronts for the generated waypoints at the same plan are given in Fig. 3 to 7 . These results show the repartition topology of the nondominated solutions through the Pareto surfaces. The best compromise solution is selected at each case thanks to the proposed TOPSIS method. These demonstrative results show high optimization performance in terms of convergence dynamics and solutions' distribution. The proposed algorithms have a good coverage of the non-dominated set of solutions that means a high variety among the different solutions of the optimization problem (5) with the considered two objective functions of Equations (2) and (3) and under operational constraints of Equation (4).

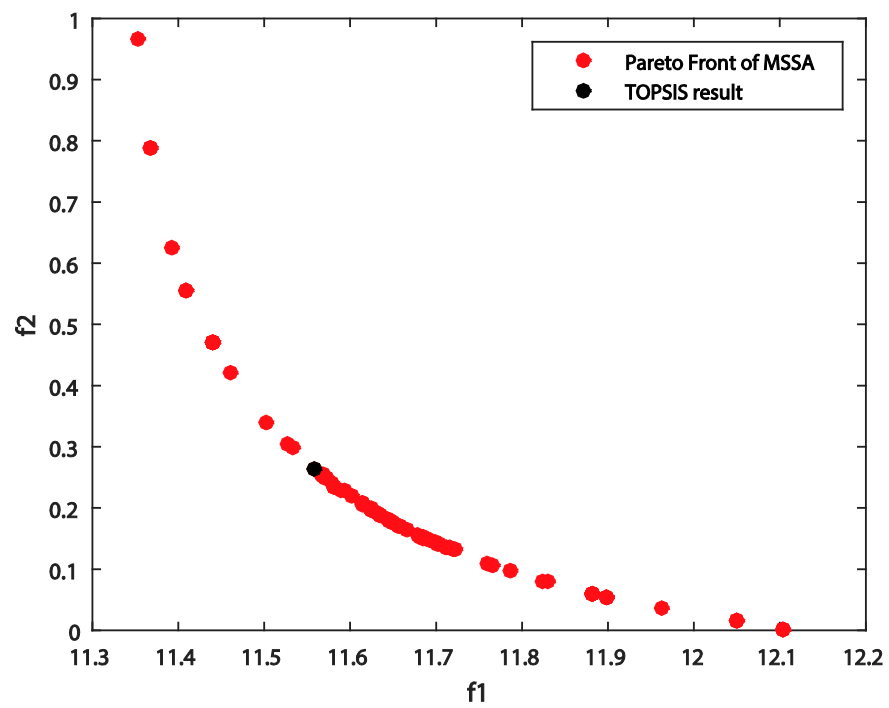

Fig. 3. Pareto Front for the Generation of a Waypoint: MSSA-based Approach.

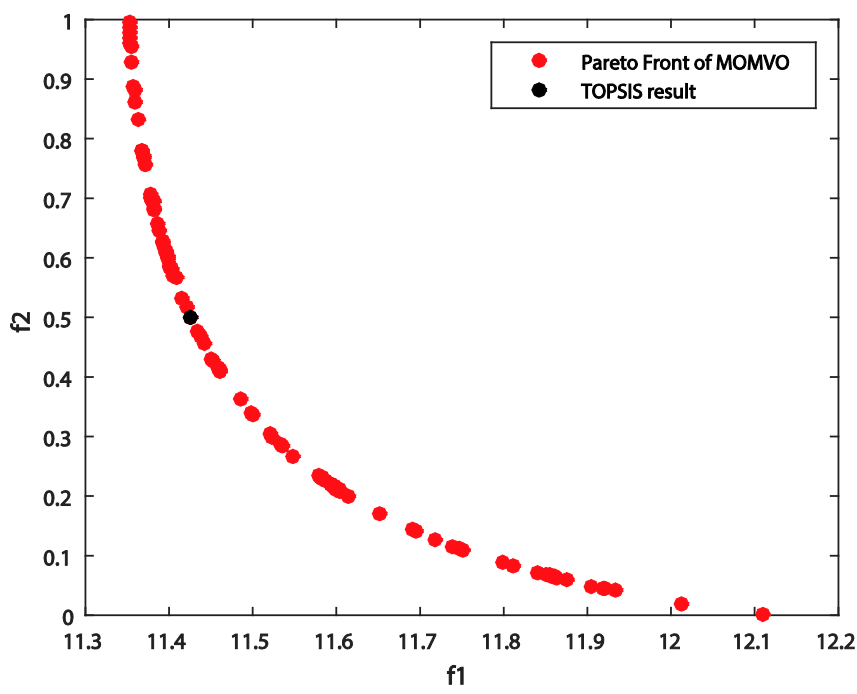

Fig. 4. Pareto Front for the Generation of a Waypoint: MOMVO-based Approach. 


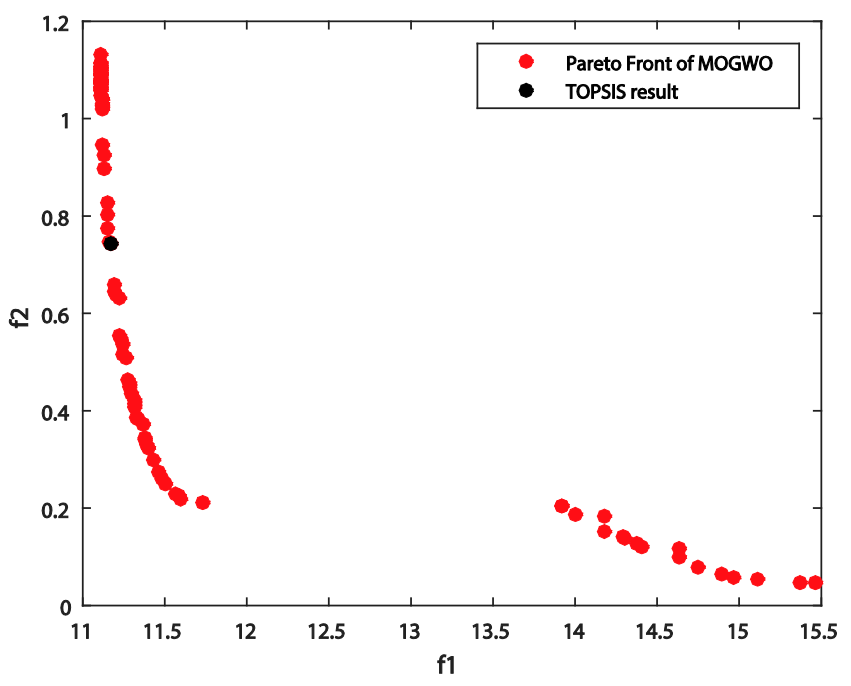

Fig. 5. Pareto Front for the Generation of a Waypoint: MOGWO-based Approach.

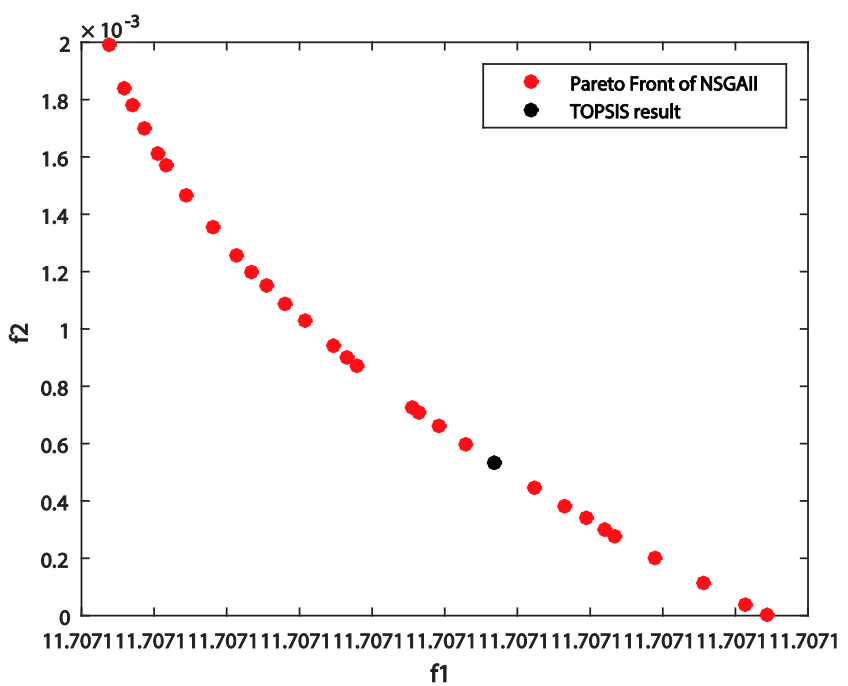

Fig. 6. Pareto Front for the Generation of a Waypoint: NSGA-II-based Approach.

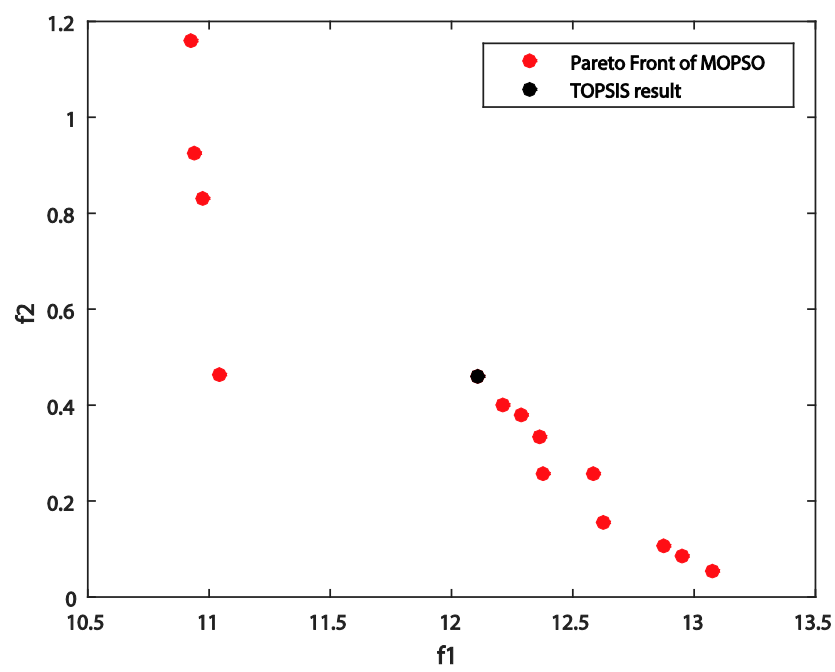

Fig. 7. Pareto Fronts for the Generation of a Waypoint: MOPSO-based Approach.
For the performance comparison purposes, various metrics such as Maximum Spread (MS) [37, 38], Hyper-Volume (HV) [39] and C-metric [40] have been employed in this study. The optimization results related to the MS-metric are presented in Table II. The average performance of the MOMVO has surpassed the other algorithms in terms of having the biggest values for the MS metrics. It may be seen that the coverage of the proposed algorithm tends to be better than other algorithms. Table III shows the comparison of the hyper-volume metrics of different methods. The MOGWO algorithm obtains the largest average of $\mathrm{HV}$ values followed by the MOMVO, which means that the MOGWO and MOMVO are the best solvers in terms of diversity and convergence performance.

The comparative results for the proposed algorithm MOMVO with others methods in terms of the C-metric are shown in Table IV. The proposed MOMVO algorithm outperformed all others competitive ones, which dominates more than $21 \%$ of the MSSA solutions, $35 \%$ of the MOGWO solutions, $1 \%$ of the NSGA-II solutions and $76 \%$ of MOPSO solutions on average. The MOGWO algorithm dominates more than $57 \%$ of the MOMVO solutions.

TABLE II. COMPARISON OF THE MS-METRIC FOR THE REPORTED ALGORITHMS

\begin{tabular}{|l|l|l|l|l|l|}
\hline & MSSA & MOMVO & MOGWO & NSGAII & MOPSO \\
\hline Best & 114.275 & 115.395 & 121.89 & 64.122 & 96.2610 \\
\hline Mean & 112.477 & 114.352 & 114.111 & 39.6856 & 63.5578 \\
\hline Worst & 111.986 & 111.745 & 113.68 & 11.7029 & 38.6300 \\
\hline STD & 1.0804 & 1.0712 & 2.23149 & 26.5066 & 28.1470 \\
\hline
\end{tabular}

TABLE III. COMPARISON OF THE HV-METRIC FOR THE REPORTED ALGORITHMS

\begin{tabular}{|l|l|l|l|l|l|}
\hline & MSSA & MOMVO & MOGWO & NSGAII & MOPSO \\
\hline Best & 0.55670 & 0.61150 & 4.22660 & $1.13 \mathrm{e}-10$ & 2.16790 \\
\hline Mean & 0.52158 & 0.59281 & 4.02710 & $2.82 \mathrm{e}-11$ & 0.54902 \\
\hline Worst & 0.47390 & 0.57180 & 3.82800 & 0.0000 & 0.29900 \\
\hline STD & 0.03270 & 0.01037 & 0.16850 & $5.65 \mathrm{e}-11$ & 0.71760 \\
\hline
\end{tabular}

TABLE IV. COMPARISON OF THE C-METRIC FOR THE REPORTED ALGORITHMS

\begin{tabular}{|l|l|l|l|l|}
\hline & Best & Mean & Worst & STD \\
\hline C (MOMVO, MSSA) & 0.34 & 0.21 & 0.09 & 0.0921 \\
\hline C (MSSA, MOMVO) & 0.04 & 0.02 & 0.00 & 0.0160 \\
\hline C (MOMVO, MOGWO) & 0.39 & 0.35 & 0.33 & 0.0254 \\
\hline C (MOGWO, MOMVO) & 0.66 & 0.57 & 0.46 & 0.0776 \\
\hline C (MOMVO, NSGAII) & 0.02 & 0.01 & 0.00 & 0.0124 \\
\hline C (NSGAII, MOMVO) & 0.25 & 0.20 & 0.14 & 0.0381 \\
\hline C (MOMVO, MOPSO) & 1.00 & 0.67 & 0.00 & 0.4306 \\
\hline C (MOPSO, MOMVO) & 0.74 & 0.28 & 0.00 & 0.3416 \\
\hline
\end{tabular}


The comparative analysis of MOMVO metaheuristic is performed with MSSA, MOGWO, NSGAII and MOPSO on three performance criteria at the five scenarios, such as the path length, the elapsed time and the 3D planned trajectory. In order to evaluate the capability of the proposed MOMVO algorithm to solve the path planning problem (5), the path length and elapsed time results on each scenario are saved over 10 simulations independently. The statistical results are summarized in Table V.

To analyze the differences between the performances of reported algorithms, nonparametric statistical tests in the sense of Friedman are implemented and discussed [41]. For the five proposed algorithms and five scenarios, the computed value of the $\chi$-distribution is equal to $\chi_{F_{1}}^{2}=10.7059$ for the path length criterion and is $\chi_{F_{2}}^{2}=46.0000$ for the elapsed time one. Based on the distribution table at a confidence level $\alpha=0.05$, the Friedman statistic, i.e. Iman-Davenport extension of the classical Friedman test, is $\chi_{0.95,4,16}^{2}=3.01<\chi_{F_{1}}^{2}<\chi_{F_{2}}^{2}$. So the null hypothesis is rejected and there are notable differences between the proposed methods for path planning problem. In order to know which algorithms are different, the post-hoc paired comparison is performed. The algorithms $i$ and $j$ are declared different when the absolute difference of the rank's sum $\left|R_{i}-R_{j}\right|$ is greater than a critical value. The summation of the ranks of different algorithms is presented in Tables VI and VII. The critical value is equal to 6.1805 for the path length criterion and is 3.3519 for the elapsed time one according to the statistic computation formula given in [42, 43]. The paired comparisons are summarized in Tables VIII and IX. The bold and underlined values in such tables designated that the performances of the reported algorithms are different.

TABLE V. Optimization Results of Problem (5)

\begin{tabular}{|c|c|c|c|c|c|c|c|c|c|c|c|}
\hline \multirow{2}{*}{\multicolumn{2}{|c|}{ Scenarios }} & \multicolumn{2}{|l|}{ MSSA } & \multicolumn{2}{|c|}{ MOMVO } & \multicolumn{2}{|c|}{ MOGWO } & \multicolumn{2}{|c|}{ NSGAII } & \multicolumn{2}{|c|}{ MOPSO } \\
\hline & & \multirow{2}{*}{\begin{tabular}{|l|}
$\begin{array}{l}\text { Path } \\
\text { length }\end{array}$ \\
11.716 \\
\end{tabular}} & \multirow{2}{*}{$\begin{array}{l}\begin{array}{l}\text { Elapsed } \\
\text { time }\end{array} \\
226.215 \\
\end{array}$} & \multirow{2}{*}{$\begin{array}{l}\begin{array}{l}\text { Path } \\
\text { length }\end{array} \\
11.633 \\
\end{array}$} & \multirow{2}{*}{$\begin{array}{l}\begin{array}{l}\text { Elapsed } \\
\text { time }\end{array} \\
179.835 \\
\end{array}$} & \multirow{2}{*}{$\begin{array}{l}\begin{array}{l}\text { Path } \\
\text { length }\end{array} \\
13.478 \\
\end{array}$} & \multirow{2}{*}{$\begin{array}{l}\begin{array}{l}\text { Elapsed } \\
\text { time }\end{array} \\
609.89 \\
\end{array}$} & \multirow{2}{*}{$\begin{array}{l}\begin{array}{l}\text { Path } \\
\text { length }\end{array} \\
10.367 \\
\end{array}$} & \multirow{2}{*}{$\begin{array}{l}\begin{array}{l}\text { Elapsed } \\
\text { time }\end{array} \\
916.874 \\
\end{array}$} & \multirow{2}{*}{$\begin{array}{l}\begin{array}{l}\text { Path } \\
\text { length }\end{array} \\
12.155 \\
\end{array}$} & \multirow{2}{*}{$\begin{array}{l}\begin{array}{l}\text { Elapsed } \\
\text { time }\end{array} \\
353.330 \\
\end{array}$} \\
\hline & Best & & & & & & & & & & \\
\hline \multirow{3}{*}{1} & Mean & 11.805 & 267.63 & 11.700 & 185.214 & 14.038 & 694.24 & 10.684 & 954.364 & 12.942 & 465.251 \\
\hline & Worst & 11.889 & 335.340 & 11.789 & 194.796 & 16.093 & 769.81 & 11.014 & 980.351 & 13.254 & 534.214 \\
\hline & STD & 0.0487 & 3.1009 & 0.0449 & 2.8908 & 1.0792 & 5.5416 & 0.0510 & 6.142 & 0.0562 & 4.354 \\
\hline \multirow{4}{*}{2} & Best & 13.906 & 463.59 & 13.814 & 313.511 & 15.776 & 740.541 & 12.160 & 1010.02 & 18.310 & 770.470 \\
\hline & Mean & 13.971 & 598.90 & 13.853 & 352.481 & 16.259 & 817.02 & 13.561 & 1100.14 & 18.563 & 820.540 \\
\hline & Worst & 14.060 & 643.75 & 13.896 & 388.173 & 16.995 & 868.63 & 14.547 & 1502.87 & 19.547 & 897.540 \\
\hline & STD & 0.0476 & 1.8751 & 0.0310 & 1.0856 & 0.3817 & 3.127 & 0.6751 & 5.3654 & 0.5541 & 3.1452 \\
\hline \multirow{4}{*}{3} & Best & 20.052 & 630.631 & 19.933 & 622.273 & 19.994 & 920.511 & 21.040 & 1246.44 & 25.035 & 859.4230 \\
\hline & Mean & 20.251 & 675.421 & 20.154 & 658.591 & 20.169 & 956.05 & 21.501 & 1424.25 & 26.584 & 898.3540 \\
\hline & Worst & 20.354 & 780.254 & 20.280 & 775.453 & 20.268 & 991.8 & 22.574 & 1914.24 & 27.984 & 950.2450 \\
\hline & STD & 0.0541 & 1.5412 & 0.0453 & 1.4516 & 0.0818 & 2.0414 & 0.1554 & 5.2541 & 0.1422 & 3.4251 \\
\hline \multirow{4}{*}{4} & Best & 22.764 & 782.680 & 22.731 & 804.85 & 23.152 & 1198.13 & 21.822 & 1584.63 & 29.273 & 1263.090 \\
\hline & Mean & 22.815 & 808.657 & 22.755 & 829.669 & 23.791 & 1257.24 & 22.783 & 2451.35 & 30.201 & 1310.250 \\
\hline & Worst & 22.867 & 889.06 & 22.804 & 845.214 & 24.213 & 1301.32 & 22.974 & 2971.35 & 31.254 & 1398.210 \\
\hline & STD & 0.0353 & 1.2592 & 0.0200 & 1.0241 & 0.0524 & 3.2145 & 0.1642 & 5.0841 & 0.1234 & 4.5412 \\
\hline \multirow{4}{*}{5} & Best & 28.953 & 974.255 & 28.883 & 950.989 & 30.218 & 2273.75 & 25.957 & 2614.11 & 34.568 & 1501.90 \\
\hline & Mean & 29.076 & 1201.35 & 28.967 & 1024.69 & 30.451 & 2468.21 & 26.874 & 2781.34 & 36.354 & 1654.21 \\
\hline & Worst & 29.354 & 2763.36 & 29.058 & 1300.53 & 30.869 & 2654.31 & 27.236 & 3594.12 & 37.541 & 1701.24 \\
\hline & STD & 0.1174 & 550.076 & 0.0530 & 112.667 & 0.3254 & 2.5471 & 0.1874 & 3.1245 & 0.1542 & 4.1243 \\
\hline
\end{tabular}

TABLE VI. AVERAge RANK ANALYSIS OF MEAN PERFORMANCES FOR THE PATH LENGTH CRITERION

\begin{tabular}{|c|c|c|c|c|c|c|c|c|c|c|c|}
\hline & \multicolumn{2}{|c|}{ Scenario 1} & \multicolumn{2}{|c|}{ Scenario 2} & \multicolumn{2}{|c|}{ Scenario 3} & \multicolumn{2}{|c|}{ Scenario 4} & \multicolumn{2}{|c|}{ Scenario 5} & \multirow{2}{*}{$\begin{array}{l}\text { Rank's } \\
\text { sum }\end{array}$} \\
\hline & Score & Rank & Score & Rank & Score & Rank & Score & Rank & Score & Rank & \\
\hline MSSA & 11.805 & 3 & 13.971 & 3 & 20.251 & 3 & 22.815 & 3 & 29.076 & 3 & 15 \\
\hline MOMVO & 11.700 & 2 & 13.853 & 2 & 20.154 & 1 & 22.755 & 1 & 28.967 & 2 & 8 \\
\hline MOGWO & 14.038 & 5 & 16.259 & 4 & 20.169 & 2 & 23.791 & 4 & 30.451 & 4 & 19 \\
\hline NSGA-II & 10.684 & 1 & 13.561 & 1 & 21.501 & 4 & 22.783 & 2 & 26.874 & 1 & 9 \\
\hline MOPSO & 12.942 & 4 & 18.563 & 5 & 26.584 & 5 & 30.201 & 5 & 36.354 & 5 & 24 \\
\hline
\end{tabular}


TABLE VII. AVERAgE RANK ANALYSIS OF MEAN PERFORMANCES FOR THE ELAPSED TIME CRITERION

\begin{tabular}{|c|c|c|c|c|c|c|}
\hline & \multicolumn{2}{|c|}{ Scenario 1} & \multicolumn{2}{|c|}{ Scenario 2} & Scena \\
\hline & & \multicolumn{2}{|l|}{ Score } & Score & Rank & Score \\
\hline \multicolumn{2}{|l|}{ MSSA } & \multicolumn{2}{|l|}{267.63} & 598.90 & 2 & 675.4 \\
\hline \multicolumn{2}{|l|}{ MOMVO } & \multicolumn{2}{|l|}{185.21} & 352.48 & 1 & 658.5 \\
\hline \multicolumn{2}{|l|}{ MOGWO } & \multicolumn{2}{|l|}{694.24} & 817.02 & 3 & 956.0 \\
\hline \multicolumn{2}{|l|}{ NSGA-II } & \multicolumn{2}{|l|}{954.36} & 1100.10 & 5 & 1424. \\
\hline MOPSO & & \multicolumn{2}{|l|}{465.25} & 820.54 & 4 & 898.3 \\
\hline \multicolumn{7}{|c|}{ TABLE VIII. } \\
\hline $\mid R_{i}-R_{j}$ & \multicolumn{2}{|c|}{ MOMVO } & MOGWO & NSGA-II & \multicolumn{2}{|c|}{ MOPSO } \\
\hline MSSA & \multicolumn{2}{|c|}{$\underline{7}$} & 4 & 6 & \multicolumn{2}{|l|}{$\underline{\mathbf{9}}$} \\
\hline MOMVO & \multicolumn{2}{|c|}{-} & $\underline{11}$ & 1 & \multicolumn{2}{|l|}{$\underline{16}$} \\
\hline MOGWO & \multicolumn{2}{|c|}{ - } & - & $\underline{10}$ & \multicolumn{2}{|l|}{5} \\
\hline NSGA-II & & - & - & - & \multicolumn{2}{|l|}{15} \\
\hline
\end{tabular}

TABLE IX. PAIRED COMPARISON OF THE PROPOSED METAHEURISTICS FOR THE ELAPSED TIME CRITERION

\begin{tabular}{|l|l|l|l|l|}
\hline$\left|R_{i}-R_{j}\right|$ & MOMVO & MOGWO & NSGA-II & MOPSO \\
\hline MSSA & 3 & $\underline{\mathbf{9}}$ & $\underline{\mathbf{6}}$ & $\underline{\mathbf{8}}$ \\
\hline MOMVO & - & $\underline{\mathbf{1 2}}$ & $\underline{\mathbf{1 9}}$ & $\underline{\mathbf{1 1}}$ \\
\hline MOGWO & - & - & $\underline{\mathbf{7}}$ & 1 \\
\hline NSGA-II & - & - & - & $\underline{\mathbf{8}}$ \\
\hline
\end{tabular}

From the results of Tables VI and VIII, i.e. comparison based on the path length criterion, the proposed MOMVO solver outperforms all the MSSA, MOGWO and MOPSO algorithms since the obtained the values of the absolute difference of the rank's sum are greater than the computed critical value 6.1805. However, such an optimizer has the same performance with the NSGA-II algorithm with an absolute difference of rank's sum equal to 1 and according to the final given by Table VI we can confirm that the MOMVO metaheuristic is better than NSGA-II in the case of path length performance metric. For the second criterion, i.e. elapsed time of Tables VII and IX, we found that the MOMVO and MSSA algorithms have the same performance but the MOMVO one is the better since it have the first final rank as given in Table VII. For the rest of competitive algorithms MOGWO, NSGA-II and MOPSO, the proposed MOMVO method has values of absolute difference greater that the computed critical value
For the defined performance criteria such as elapsed time, path length and threats avoidance, the planned paths are shown in Fig. 8, 9 and 10 for only the hard flight situations, i.e. $3^{\text {rd }}, 4^{\text {th }}$ and $5^{\text {th }}$ flight scenarios with an increasing number of threats. These curves correspond to the optimization results for the mean case. As observed in these figures, the MSSA, MOMVO and MOGWO competitive metaheuristics give the most direct path. They are perfect in all scenarios and can avoid all obstacles and threats, which ensure their high efficiency in flight planning. The path obtained by the MOPSO method avoids all obstacles but takes a long distance in comparison with others algorithms. The NSGA-II algorithm gives a direct path but with a very low level of flight in the case of scenarios 1, 2 and 5 as shown in Fig. 10. The planned path for this solver passes through an obstacle zone in scenarios 3 and 4 as depicted in Fig. 8 and Fig. 9. So, it is too difficult to take into account all the obstacles in certain scenarios.

\section{Algorithms' Sensitivity Analysis}

In this section, the performance of the two considered main indicators, i.e. path length and execution time, is analyzed with the variations in the population size and iterations values of the competitive algorithms. The performance comparison is given under the $2^{\text {nd }}$ scenario. The results are presented in Tables $\mathrm{X}$ and XI. Keeping the iterations constant, the path length decreases linearly with the augment of the population size for all algorithms, on the contrary, the execution time increases. When the population size is constant, the elapsed time varies with the iterations' numbers on the contrary the path length is shorter. The proposed MOMVO algorithm remains robust under these variations and clearly outperforms all others proposed solvers with the shortest path and the minimum elapsed time in most cases. This main capability makes the proposed MOMVO algorithm more adapted for path planning problems. 
TABle Xi. Path Length VARiation under Iterations and Population Size Parameters of Problem (6)

\begin{tabular}{|c|c|c|c|c|c|c|}
\hline \multirow{2}{*}{ Generation } & \multirow{2}{*}{ Population size } & \multicolumn{5}{|c|}{ Path length (km) } \\
\hline & & MSSA & MOMVO & MOGWO & NSGAII & MOPSO \\
\hline \multirow{3}{*}{50} & 20 & 16.0348 & 13.8620 & 16.4859 & 15.0695 & 62.7104 \\
\hline & 50 & 13.9310 & 13.8593 & 17.0208 & 13.9520 & 31.8837 \\
\hline & 100 & 13.8869 & 13.8411 & 18.9750 & 13.8508 & 20.2795 \\
\hline \multirow{3}{*}{100} & 20 & 15.0420 & 13.8603 & 18.4772 & 14.8723 & 29.9692 \\
\hline & 50 & 13.9440 & 13.8549 & 17.0723 & 13.8661 & 23.4170 \\
\hline & 100 & 13.8354 & 13.8235 & 15.8578 & 13.8421 & 20.6154 \\
\hline \multirow{3}{*}{200} & 20 & 14.1662 & 13.8602 & 16.9149 & 14.5471 & 19.5114 \\
\hline & 50 & 13.9049 & 13.8509 & 16.9512 & 13.8574 & 20.8871 \\
\hline & 100 & 13.8244 & 13.8226 & 15.8345 & 13.8314 & 19.6975 \\
\hline
\end{tabular}

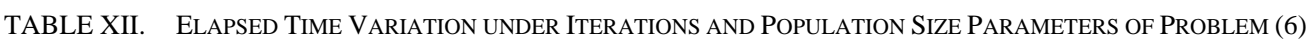

\begin{tabular}{|c|c|c|c|c|c|c|}
\hline \multirow{2}{*}{ Generation } & \multirow{2}{*}{ Population size } & \multicolumn{5}{|c|}{ Elapsed Time (sec) } \\
\hline & & MSSA & MOMVO & MOGWO & NSGAII & MOPSO \\
\hline \multirow{3}{*}{50} & 20 & 57.3806 & 56.7261 & 179.4049 & 583.8650 & 279.4850 \\
\hline & 50 & 141.9460 & 127.4121 & 371.6397 & 2851.5570 & 533.2424 \\
\hline & 100 & 248.4768 & 279.4000 & 794.6620 & 4741.3340 & 1192.1590 \\
\hline \multirow{3}{*}{100} & 20 & 74.5709 & 100.4210 & 574.5162 & 610.6982 & 126.6340 \\
\hline & 50 & 176.4819 & 168.6157 & 638.9724 & 3294.8690 & 1336.0780 \\
\hline & 100 & 365.5790 & 277.4627 & 1030.1680 & 5241.4120 & 3628.1100 \\
\hline \multirow{3}{*}{200} & 20 & 150.4783 & 199.9634 & 432.3340 & 784.2150 & 1949.6250 \\
\hline & 50 & 424.0723 & 420.8711 & 1121.6300 & 4145.1420 & 3980.8930 \\
\hline & 100 & 936.5628 & 654.8361 & 2156.1890 & 5987.1240 & 4413.2450 \\
\hline
\end{tabular}
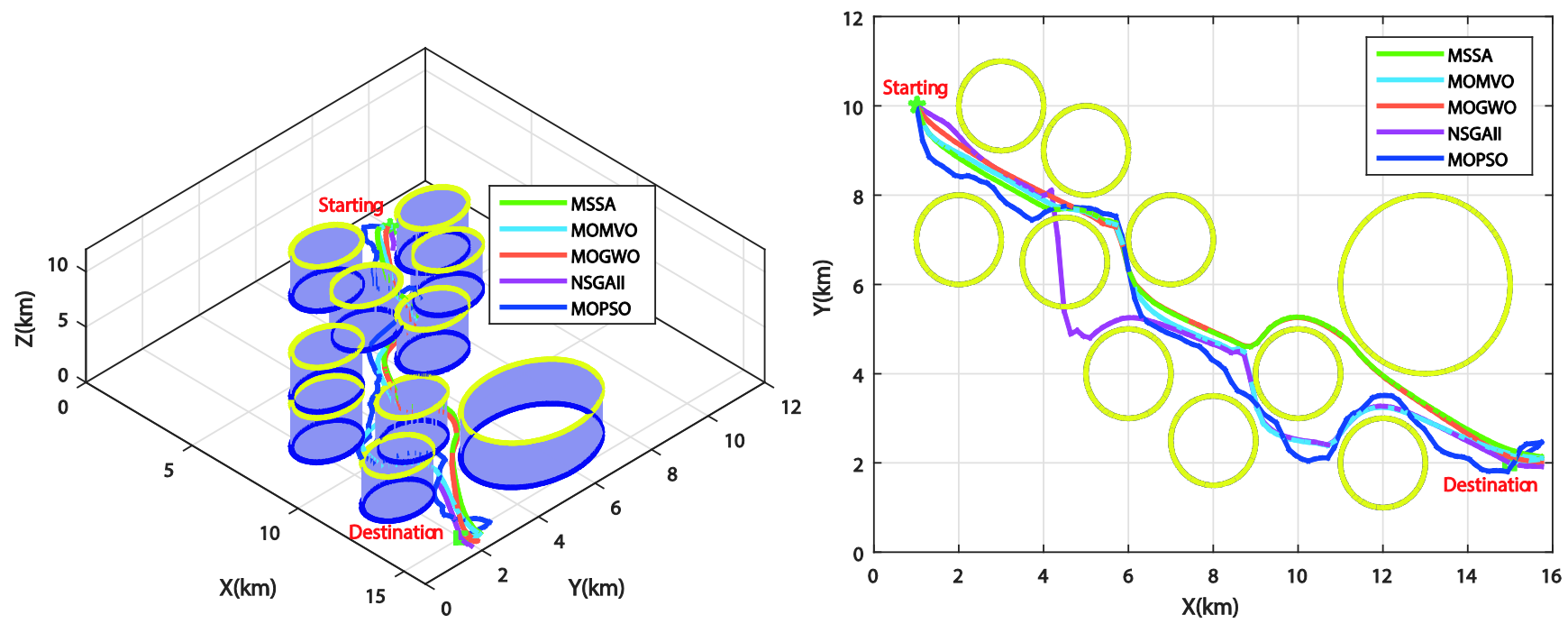

Fig. 8. Performance Comparisons in $3^{\text {rd }}$ Scenario: 10 Threats' Avoidance. 

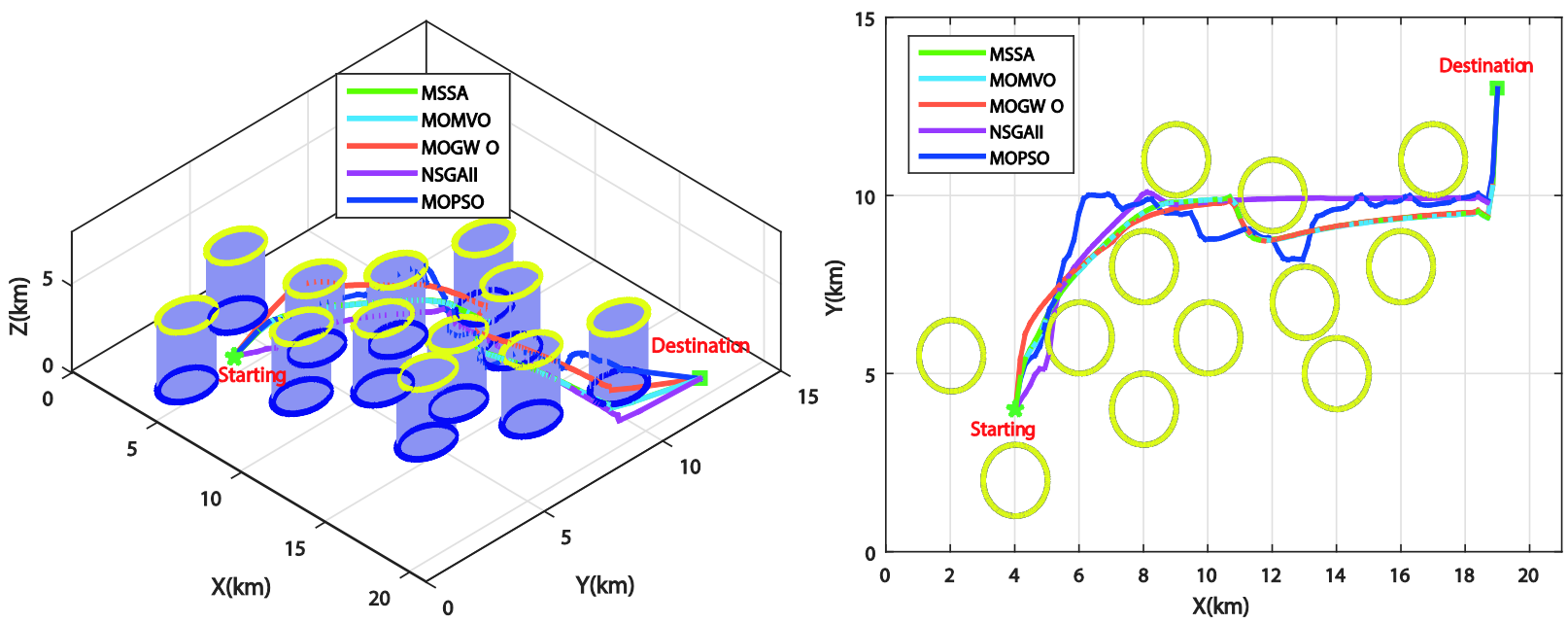

Fig. 9. Performance Comparisons in $4^{\text {th }}$ Scenario: 12 Threats' Avoidance.
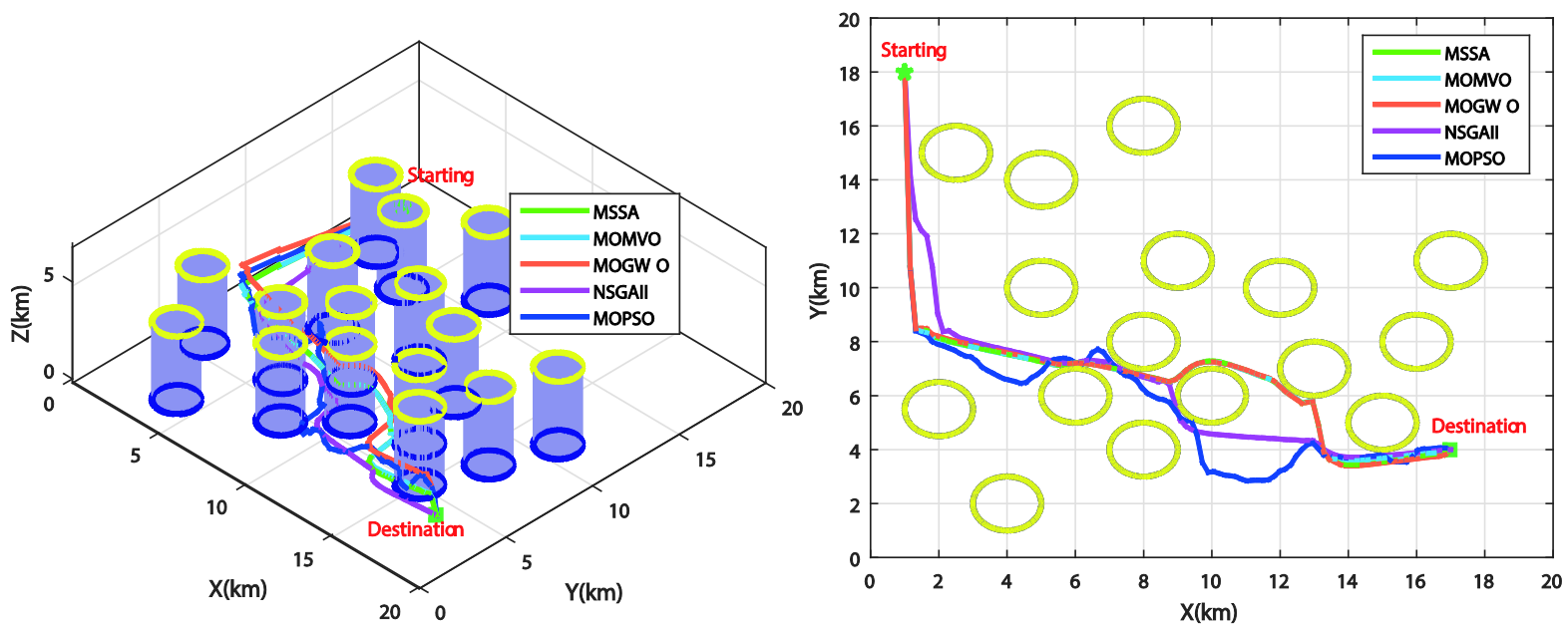

Fig. 10. Performance Comparisons in $5^{\text {th }}$ Scenario: 16 Threats' Avoidance.

\section{CONCLUSION}

In this paper, the path planning problem for unmanned aerial vehicles is reformulated by transforming the generation of each flight waypoint into a constrained multiobjective optimization problem. An offline path planning algorithm has been developed and applied within various flight scenarios containing an increasing number of the threats and static obstacles. For an efficient resolution of the formulated multiobjective optimization problem, a recent variant of the MOMVO metaheuristic has been proposed and successfully implemented. A set of competitive algorithms such as MSSA, MOGWO, MOPSO and NSGA-II are retained throughout the study as performance comparison tools. An empirical study of these algorithms has been firstly performed for solving different multi-criteria test functions from the literature. The proposed MOMVO algorithm outperforms all others reported methods in the majority of test functions cases as well as for the real path planning formulated problem. The demonstrative simulations as well as the nonparametric Friedman and paired comparison tests show the effectiveness and superiority of the proposed TOPSIS and MOMVO-based path planning approach in comparison with the reporter competitive algorithms. To further demonstrate the performance of the proposed MOMVO algorithm, several metrics and criteria are employed such as the elapsed time, the path length and threats avoidance capability. The simulation results and comparisons show that the proposed algorithms can successfully solve the 3D UAVs' path planning problem with a remarkable superiority of the MOMVO-based approach. Other improvements should be made in future works as the extension to the paths planning of cooperative multiUAVs as well as the flight in an environment with dynamic obstacles.

\section{REFERENCES}

[1] A. Idalene, K. Boukhdir and H. Medromi, "UAV Path Planning for Civil Applications”, Int. J. of Adv. Comp. Sci. and Appli (IJACSA)., vol. 10, no. 12, pp. 635-641, 2019.

[2] S.J. Chung, A. Paranjape, P. Dames, S. Shen and V. Kumar, "A survey on aerial swarm robotics", IEEE Trans. on Rob., vol. 34, no. 4, pp. 837855, 2018.

[3] X. Chen, X. Chen and G. Xu, "The path planning algorithm studying about UAV attacks multiple moving targets based on Voronoi diagram," Int. J. of Cont. and Autom., vol. 9, no. 1, pp. 281-292, 2016.

[4] C. A. Cindy and S. O. Rebeca, "Swarm Robotics and Rapidly Exploring Random Graph Algorithms Applied to Environment Exploration and Path Planning”, Int. J. of Adv. Comp. Sci. and Appli. (IJACSA), vol. 10, no. 5, pp. 692- 702, 2019. 
[5] R. Tianzhu, Z. Rui, X. Jie and D. Zhuoning, "Three-dimensional path planning of UAV based on an improved A* algorithm,” In Proc. of IEEE Chinese Guid., Navig. and Cont. Conf. (CGNCC), pp. 140-145, Nanjing, China, August 12-14, 2016.

[6] H. Kim, J. Jeong, N. Kim and B. Kang, "A Study on 3D Optimal Path Planning for Quadcopter UAV Based on D* Lite,” In Proc. of the Int. Conf. on Unm. Air. Syst. (ICUAS), pp. 787-793, Atlanta, GA, USA, June 11-14, 2019.

[7] I. Iswanto, M. Alfian, W. Oyas and I.C. Adha, "Artificial Potential Field Algorithm Implementation for Quadrotor Path Planning” Int. J. of Adv. Comp. Sci. and Appli. (IJACSA), vol. 10, no. 8, pp. 575 - 585, 2019.

[8] Y.G. Fu, M.Y. Ding, C.P. Zhou and H.P. Hu, "Route planning for unmanned aerial vehicle on the sea using hybrid differential evolution and quantum-behaved particle swarm optimization”, IEEE Trans. on Syst., Man, and Cyb. : Syst., vol. 43, no. 6, pp. 1451-1465, 2013.

[9] A. Gümüşçü, M. Tenekeci and A. Tabanlığlu, The shortest path detection for unmanned aerial vehicles via genetic algorithm on aerial imaging of agricultural lands. Int. Adv. Res. and Eng. J., vol. 2, no. 3, pp. 315-319, 2018.

[10] D. M. Vijayakumari, S. Kim, J. Suk and H. Mo, "Receding-horizon trajectory planning for multiple UAVs using particle swarm optimization”, In Proc. of AIAA Scitech 2019 Forum , pp. 1165, San Diego, California, January 7-11, 2019.

[11] B. Abhishek, S. Ranjit, T. Shankar, G. Eappen, P. Sivasankar and A. Rajesh, "Hybrid PSO-HSA and PSO-GA algorithm for 3D path planning in autonomous UAVs,” SN App. Sci., vol. 2, no. 11, pp. 1-16, 2020.

[12] R.K. Dewangan, A. Shukla and W.W. Godfrey, "Three dimensional path planning using Grey wolf optimizer for UAVs”, App. Intelligence, vol. 49, no. 6, pp. 2201-2217, 2019.

[13] B. Li, X. Qi, B. Yu and L. Liu, "Trajectory Planning for UAV Based on Improved ACO Algorithm,” IEEE Access, vol. 8, pp. 2995-3006, 2019.

[14] Y. Chen, J. Yu, Y. Mei, Y. Wang and X. Su, "Modified central force optimization (MCFO) algorithm for 3D UAV path planning", Neurocomputing, vol. 171, pp. 878-888, 2015.

[15] Y. Liu, X. Zhang, X. Guan and D. Delahaye, "Sensitivity decision based path planning algorithm for unmanned aerial vehicle with improved particle swarm optimization”, Aero. Sci. and Tech., vol. 58, pp. 92-102, 2016.

[16] P.K. Das, H.S. Behera and B.K. Panigrahi, "A hybridization of an improved particle swarm optimization and gravitational search algorithm for multi-robot path planning”, Swa. and Evol. Comp., vol. 28, pp. 14-28, 2016.

[17] R. Madiouni, S Bouallègue, J. Haggège and P. Siarry, "EpsilonMultiobjective Particle Swarm Optimization Based Tuning of Sensitivity Functions for RST Digital Control Design”, Trans.. of the Inst. of Meas. and Cont. (TIMC), vol. 41, no. 13, pp. 3688-3704, 2019.

[18] R. Madiouni, S. Bouallègue, J. Haggège and P. Siarry, "Robust RST Control Design based on Multi-objective Particle Swarm Optimization Approach”, Int. J. of Cont., Aut., and Syst. (IJCAS), vol. 14, no. 6, pp. 1607-1617, 2016.

[19] C. Ramirez-Atencia, G. Bello-Orgaz, M. D. R-Moreno and D. Camacho, "Solving complex multi-UAV mission planning problems using multiobjective genetic algorithms", Soft Comp., vol. 21, no. 17, pp. 48834900, 2017.

[20] X. Zhen, Z. Enze and C. Qingwei, "Rotary unmanned aerial vehicles path planning in rough terrain based on multi-objective particle swarm optimization”, J. of Syst. Eng. and Elec.,vol.31,no. 1, pp. 130-141, 2020.

[21] J. Liu, W. Wang, X. Li, T. Wang, S. Bai and W. Yanfeng, "Solving a multi-objective mission planning problem for UAV swarms with an improved NSGA-III algorithm,” Int. J. of Comp. Intel. Syst., vol. 11, no. 1, pp. 1067-1081, 2018.

[22] Z. Wang, M. Li, L. Dou, Y. Li, Q. Zhao and J. Li, "A novel multiobjective artificial bee colony algorithm for multi-robot path planning," In Proc. of Int. Conf. on Info. and Autom. , pp. 481-486, 2015, Lijiang, China , August 8-10, 2015.

[23] Q. Ren, Y. Yao, G. Yang and X. Zhou, "Multi-objective Path Planning for UAV in the Urban Environment Based on CDNSGA-II", In Pro. of 2019 IEEE Int. Conf. on Ser.-Ori. Syst. Eng., pp. 350-3505, April 2019.
[24] C.R. Atencia, J. Del Ser and D. Camacho, "Weighted strategies to guide a multi-objective evolutionary algorithm for multi-UAV mission planning”, Swa. and Evo. Comp., vol. 44, pp. 480-495, 2019.

[25] S. Thabit and A. Mohades, "Multi-Robot Path Planning Based on Multi-Objective Particle Swarm Optimization”, IEEE Access, vol. 7, pp. 2138-2147, 2019.

[26] Y. Yang, J. Liu, S. Tan, and H. Wang, “A multi-objective differential evolutionary algorithm for constrained multi-objective optimization problems with low feasible ratio", Applied Soft Computing, vol. 80, pp. 42-56, 2019.

[27] D.A.G.. Vieira, R. Adriano, L.A. de Vasconcelos, "Handling constraints as objectives in a multiobjective genetic based algorithm", J. of Micro., Opto. and Elec. Appli. (JMOe), vol. 2, no. 6, pp. 50-58, 2002.

[28] C.L. Hwang and K..Yoon, Multiple Attribute Decision Making: Methods and Applications: a State-of-art Survey. Springer-Verlag, New York, 1981.

[29] Z. K. Feng, S. Liu, W. J. Niu, Z. Q. Jiang, B. Luo, and S. M. Miao, "Multi-objective operation of cascade hydropower reservoirs using TOPSIS and gravitational search algorithm with opposition learning and mutation”. Water, vol. 11, no. 10, pp. 2040, 2019.

[30] S. Mirjalili, S.M. Mirjalili and A. Hatamlou, "Multi-verse optimizer: a nature-inspired algorithm for global optimization”, Neur. Comp. and Appli., vol. 27, pp. 495-513, 2016.

[31] S. Mirjalili, P. Jangir, S.Z. Mirjalili, S. Saremi and I.N. Trivedi, "Optimization of problems with multiple objectives using the multiverse optimization algorithm”, Know. Bas. Syst., vol. 134, pp. 50-71, 2017.

[32] Q. Zhang, A. Zhou, S. Zhao, P. N. Suganthan, W. Liu and S. Tiwari Multiobjective optimization test instances for the CEC 2009 special session and competition. Technical Report CES-487, University of Essex, Nanyang Technological University, (2009).

[33] S.A. Mirjalili, A.H. Gandomib, S.Z. Mirjalilic, S. Saremia, H. Farisd, and S.M. Mirjalili, "Salp Swarm Algorithm: a bio-inspired optimizer for engineering design problems”, Adv. in Eng. Soft., vol. 114, no. 12, pp. 163-191, 2017.

[34] S. Mirjalili, S.M. Mirjalili, S. Saremi and L. Coelho, "Multiobjective grey wolf optimizer: A novel algorithm for multicriterion optimization", Exp. Syst. with Appli., vol. 47, pp. 106-119, 2016.

[35] K. Deb, A. Pratap, S. Agarwal and T. Meyarivan, "A fast and elitist multi-objective genetic algorithm: NSGAII”, IEEE Trans. on Evol. Comp., vol. 6, no. 2, pp. 182-190, 2002.

[36] C.A.C. Coello, G.T. Pulido and M.S. Lechuga, "Handling multiple objectives with particle swarm optimization", IEEE Trans. on Evol. Comp., vol. 8, no. 3, pp. 256-260, 2004.

[37] E. Zitzler, K. Deb and L. Thiele, "Comparison of multiobjective evolutionary algorithms: Empirical results”, Evol. Comp., vol. 8, no. 2, pp. 173-195, 2000.

[38] S. Khalilpourazari, B. Naderi and S. Khalilpourazary, "Multi-Objective Stochastic Fractal Search: a powerful algorithm for solving complex multi-objective optimization problems”. Soft Computing, vol. 24, no. 4, pp. 3037-3066, 2020.

[39] E. Zitzler, L. Thiele, M. Laumanns, C.M. Fonseca and V.G.D. Fonseca, "Performance assessment of multiobjective optimizers: an analysis and review”, IEEE Trans. on Evol. Comp., vol. 7, no. 2, pp. 117-132, 2003.

[40] E, Zitzler and L, Thiele, "Multiobjective evolutionary algorithms: a comparative case study and the strength Pareto approach", IEEE Trans. on Evol. Comp., vol. 3, no. 4, pp. 257-271, 1999.

[41] W.J. Conover, Practical Nonparametric Statistics, 3rd edition, John Wiley \& Sons, New York, 1999.

[42] M. Mazen Alhato, S. Bouallègue, "Thermal exchange optimization based control of a doubly fed induction generator in wind energy conversion systems,” Ind. J. of Elect. Eng. and Comp. Sci. (IJEECS), vol. 20, no. 3, pp. 1252-1260, 2020.

[43] M. Mazen Hato, S. Bouallègue, "Whale Optimization Algorithm for Active Damping of LCL-Filter-based Grid-Connected Converters", Int. J. of Ren. Ener. Res. (IJRER), vol. 9, no. 2, pp. 986-996, 2019. 